\title{
A clinical, psychophysical, and electroretinographic survey of patients with autosomal dominant retinitis pigmentosa
}

\author{
A L LYNESS, W ERNST, M P QUINLAN, G M CLOVER, G B ARDEN, \\ R M CARTER, A C BIRD, AND J A PARKER
}

From the Departments of Clinical Ophthalmology and Visual Science, Institute of Ophthalmology and Moorfields Eye Hospital, London

SUMMARY We have surveyed 104 patients (44 families) with autosomal dominant retinitis pigmentosa. The range of the survey includes clinical history, ocular examination, documentation of genetic history, Goldmann kinetic perimetry with IV/4 and I/4 white targets, two-colour static perimetry, and scotopic and photopic electroretinography. Comparison of interfamilial and intrafamilial patterns in the static perimetry data strongly suggests there may be at least two genetic subgroups within the disease characterised by the pattern of loss of rod function: in subgroup D (13 patients, 4 families) this is diffuse and severe, while in subgroup $R$ ( 28 patients, 13 families) it is regional. In both $\mathrm{D}$ and $\mathrm{R}$ loss of cone function is regional, and in $\mathrm{R}$ it coincides with loss of rod function. In $D$ patients the rod electroretinogram is absent; in all but two $R$ cases it is present and usually substantial. All D patients were aware of night blindness before the age of 10 , but most $R$ patients not until after the age of 20. Many of the patients could not be classified because their disease was so advanced. The effect of disease duration on visual acuity and visual field area is described for all patients.

Retinitis pigmentosa (RP) is a term used to denote a group of disparate, genetically determined disorders characterised clinically by nyctalopia, progressive loss of visual field, a characteristic appearance of the fundus, and preservation of good visual acuity until late in the disease process. So far a clearly defined subdivision has not been devised except on genetic grounds. A better subclassification would help in advising the patient and is required if research into pathogenesis or treatment of these disorders is to be successful.

The mode of inheritance of RP may be autosomal recessive (AR), autosomal dominant (AD), or Xlinked (X-L). There is, however, evidence of heterogeneity within the genetic subgroups. Krill' suggested that division of AR disease into those with early onset and those with late onset may be helpful. Berson $e t$ $a l .{ }^{2}$ suggested that AD RP may be divided into two groups on the basis of gene penetrance (namely, full and reduced), and stated that the temporal character-

Correspondence to W Ernst, Department of Visual Science, Institute of Ophthalmology, Judd Street, London WC1H 9QS. istics of the electroretinogram differ between the two groups. Further subdivision of both simplex and AD RP has been suggested by Massof and Finkelstein ${ }^{3}$ based on different relationships between rod and cone sensitivity as determined by two-colour static perimetry on the dark adapted eye. These authors ${ }^{4}$ have developed their classification for AD RP and have also noted differences in the age of onset of night blindness between the two subtypes they propose. Other authors have proposed subgroups on the basis of electroretinographic data. ${ }^{56}$

Heterogeneity within a genetic group may be explained by the effects of different genes, which, if proved, would render subdivision helpful to research in that each subgroup would represent a pure sample of one disease. However, subdivision would be unhelpful if the different characteristics were due to observation of the same disorder at different stages of evolution or different phenotypic expression of the same genetic abnormality.

In the present study our main objective was to determine whether we could define reliable criteria 
by which AD RP could be subdivided. We have therefore surveyed a large number of patients with this inheritance pattern. Where possible, large family units were examined. Our approach was to attempt to characterise the disease within a family and then to compare interfamilial and intrafamilial variation. We used the techniques of electrophysiology, static perimetry, Goldmann kinetic perimetry, and clinical examination.

\section{Patients and methods}

\section{PATIENTS}

Affected members from families with autosomal dominant retinitis pigmentosa were invited to take part in this study; 104 patients were examined, ranging in age from 11 to 77 years. The age and sex distribution of the patients is shown in Table 1. As many affected family members as possible were recruited, and the genetic histories were verified with the patients. AD RP can be diagnosed with confidence where there is transmission through three generations, equal male and female involvement, and male to male inheritance. The diagnosis was, however, assumed where there were two or more identified affected generations, with equal severity of disease in males and females, but in the absence of male to male inheritance. Table 2 gives genetic details of the families.

Among patients with signs of RP and AD inheritance we have seen those whose fundus changes are restricted to a small area of retina, usually in the

Table 1 Age and sex distribution of the patients studied

\begin{tabular}{lcc}
\hline $\begin{array}{l}\text { Age groups } \\
\text { (years) }\end{array}$ & Male & Female \\
\hline $0-9$ & 0 & 0 \\
$10-19$ & 5 & 9 \\
$20-29$ & 11 & 10 \\
$30-39$ & 6 & 20 \\
$40-49$ & 7 & 13 \\
$50-59$ & 4 & 9 \\
$60-69$ & 5 & 4 \\
$70-79$ & 1 & 0 \\
Total & 39 & 65 \\
\hline
\end{tabular}

inferior, nasal quadrant. Though the functional changes may be more widespread, they are usually minimal in the area of retina which has a normal appearance. A comparison of family members of various ages suggests that there is no marked progress of the disease outside the area of retinal change. Such cases, commonly described as sector RP, are considered to be a distinct form of disease and have not been included in the present survey.

\section{CLINICAL EXAMINATION}

A clinical history was taken with specific questions being asked about (1) the age of onset of night blindness, (2) the age of onset of loss of visual field, and (3) the age of onset of any loss of visual acuity which could not be corrected with glasses. The visual acuity of each eye was measured after correction of any refractive error. Slit-lamp examination and examination of the fundus by indirect ophthalmoscopy and Hruby lens were performed routinely. A note was made of the presence or absence of lens opacities and their type when present, and the presence or absence of vitreous cells. The following attributes of the fundus were recorded: disc colour, retinal vessel changes, pigment changes at the level of the pigment epithelium and neurosensory retina, the distribution of changes, and the presence or absence of retinal oedema. Colour photography and fluorescein angiography were performed on most of the patients.

\section{SPECIAL EXAMINATIONS}

Visual fields were recorded with a calibrated Goldmann perimeter using the IV/4 and I/4 white targets. Upper field (above the horizontal meridian) and lower field (below the horizontal meridian) areas were measured by placing transparent graph paper over the Goldmann charts and measuring in $\mathrm{cm}^{2}$ the areas enclosed by the respective contours.

All patients were examined with a two-colour, automated, static perimeter which consists of a rotatable Lister arm in which the targets have been replaced by two light emitting diodes (LEDs) suitably filtered to emit green light of dominant wavelength $530 \mathrm{~nm}$ and red light of dominant wavelength

Table 2 Genetic details of patients

\begin{tabular}{|c|c|c|c|c|c|c|}
\hline & \multicolumn{3}{|c|}{ Male to male } & \multicolumn{3}{|c|}{ No male to male } \\
\hline & 2 Gen & 3 Gen & $>3$ Gen & 2 Gen & 3 Gen & $>3 \mathrm{Gen}$ \\
\hline Families & 3 & 9 & 6 & 8 & $11(1)$ & $7(1)$ \\
\hline Males & 3 & 12 & 10 & 6 & 8 & 0 \\
\hline Females & 1 & 8 & 14 & 17 & $11(1)$ & $14(2)$ \\
\hline
\end{tabular}

Numbers in brackets refer to families with incomplete penetrance or to individuals from such families. Gen=generation. 
$660 \mathrm{~nm}$. The light intensity can be varied in steps of $0 \cdot 1 \log$ unit over a range of $5 \log$ units. The maximum luminance of the filtered green LED is $0.9 \log \mathrm{cd} \mathrm{m}^{-2}$ and that of the filtered red is $2 \cdot 2 \log \mathrm{cd} \mathrm{m}^{-2}$. The perimeter is linked to a computer and visual thresholds can be measured at various locations in the visual field, as determined by a preset computer program; the results are stored immediately. Thresholds are usually expressed as the logarithm of the ratio of the patient's reading to the mean normal value based on data collected from a minimum of 15 observers. $^{7}$

The use of two colours, one green and one red, permits an assessment of the relative sensitivity of the rod and cone mechanisms in a particular region of the retina. Disturbance of rod function is identified by a threshold elevation for the green test light and of cone function by a threshold elevation for the red test light. The difference between the logarithms of these two values (subsequently referred to as the G-R index) is a measure of the degree to which rod function is more selectively disturbed than cone function. To a first approximation, when the difference is near zero, the disturbance is affecting cone vision to the same or greater extent than rod vision. When the difference assumes a significant positive value, then rod vision is being disturbed to a greater extent. The interpretation of the threshold data is discussed in more detail by Ernst $e t$ al. ${ }^{\prime}$

The technique used for electroretinography is described by Arden et al. ${ }^{\text {8 }}$ Gold foil electrodes were used and electroretinograms (ERGs) evoked by a variety of ganzfeld stimuli. Of particular interest were responses obtained from blue flashes, which in normal observers tended to produce a saturated rod $b$ wave (run 12, Arden et al. ${ }^{8}$ ) and red flashes which gave an ERG with a distinct cone b wave (run 18).

\section{Results}

\section{STATIC PERIMETRY}

For static perimetry the majority of patients were tested at 30 to 40 locations on four meridians $\left(0^{\circ}, 60^{\circ}\right.$, $180^{\circ}, 315^{\circ}$ ) in the right eye (see Ernst et al..$\left.^{7}\right)$. To describe the main features of a patient's field losses we have found it sufficient to display results taken from the horizontal meridians $\left(0^{\circ}\right.$ and $\left.180^{\circ}\right)$.

Fig. 1 (upper) shows a patient's thresholds (crosses) for the green target on the horizontal meridian, together with mean normal values for the same locations (asterisks). These normal means are subtracted from the patient's data to give log relative thresholds (Fig. 1, lower). To reduce the data further we have divided the meridians into five zones as shown in Fig. 1 (lower); data from the $20^{\circ}-40^{\circ}$ and $50^{\circ}-70^{\circ}$ zones have been averaged. The result from each zone is indicated by a particular symbol-for example, a square represents a value for a $10^{\circ}$ eccentricity in the temporal field. Data obtained with the red target have been processed in a similar manner.

To permit comparison between patients we have rearranged the data from any one patient (e.g., Fig. 1 , lower) so that log relative thresholds from the five zones appear on one vertical axis with a solid line linking the extreme values; data from other patients can then be presented alongside (Fig. 2A).

Illustrative data from four families are presented in Figs. 2A, B, C. There was a close similarity between the pattern of results shown by individuals in the same family. These four families also displayed two contrasting patterns. In families 30 and 225 the log relative thresholds for green were high throughout
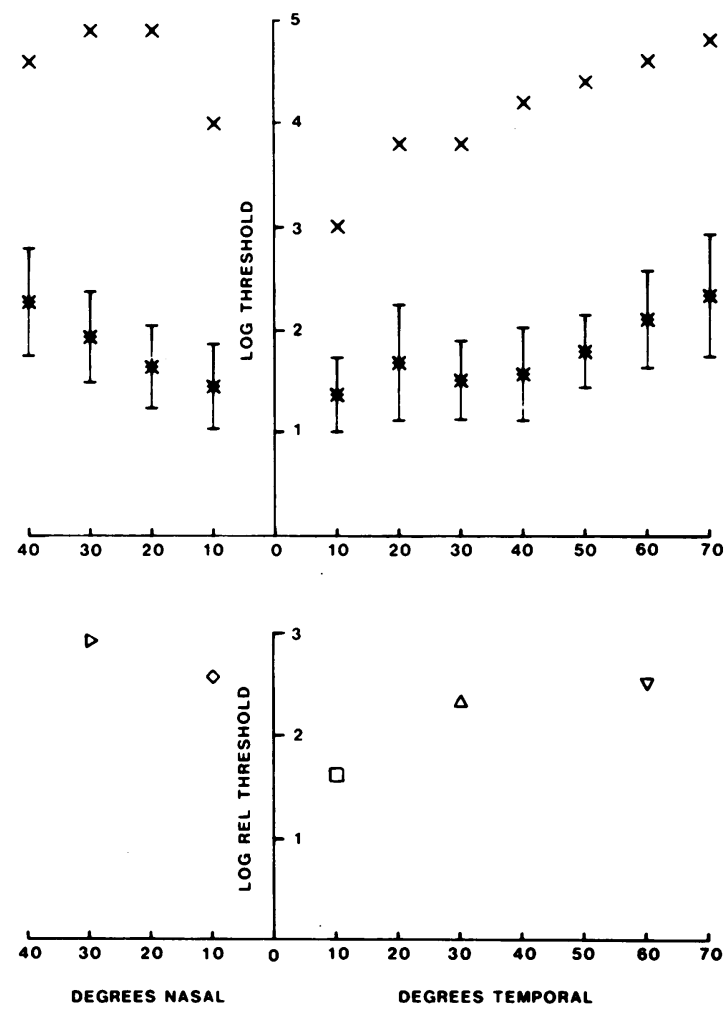

Fig. 1 Upper: Log threshold for the green target on the horizontal meridian. Crosses $=$ data from patient 30/74. Asterisks=normal means. Bars =standard deviations. Lower: Patient's data from Fig. 1 (upper) transformed to give log threshold values relative to normal in five zones: $20-40^{\circ}$ nasal (right-pointing triangle); $10^{\circ}$ nasal (diamond); $10^{\circ}$ temporal (square); 20-40 temporal (upward-pointing triangle); and 50-70 temporal (downward-pointing triangle). Values in the $20-40^{\circ}$ zones and the $50-70^{\circ}$ zone averaged. 
GREEN
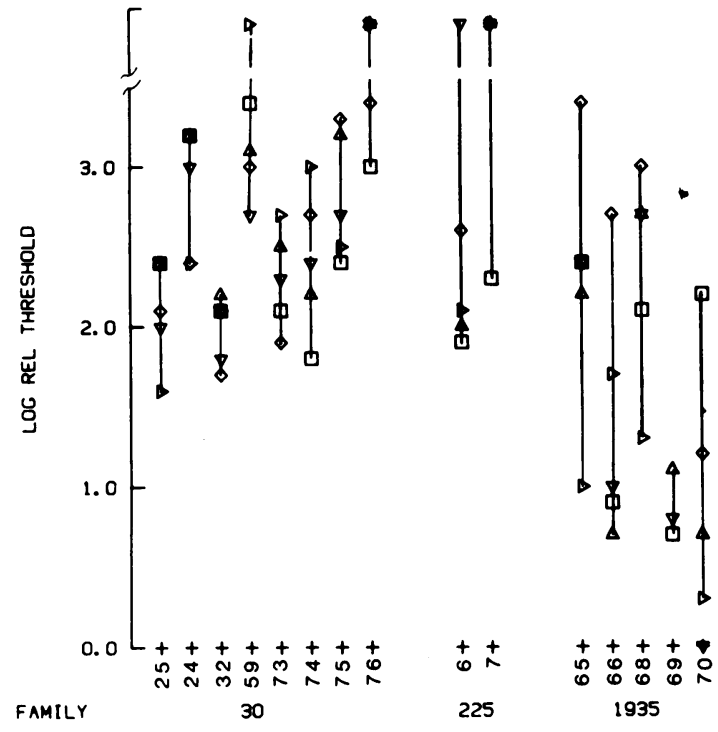

1935

the visual field, and thresholds at no point lay within $1.5 \log$ units of normal (Fig. 2A). Families 1935 and 479 , however, showed a wide range of readings for green extending from near normal to 'off scale' for different locations. Thus in families 30 and 225 there was severe and widespread loss of rod function, whereas in families 1935 and 479 there were some zones with severe rod disturbance, but there was at least one zone with near normal rod function.

The four families demonstrated a spread of read-

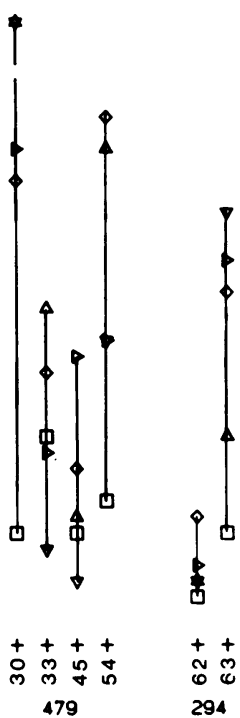

Fig. 2A Log relative thresholds for the green target in the five zones placed on a vertical axis. Axis broken to accommodate off-scale readings. Symbols as for Fig. 1 (lower). Lines link extreme values. Data from four families, 30, 225, 1935 , and 479, are presented and individual members are identified by separate numbers arranged horizontally.

ings for the red target from those indicating near normal cone function to those indicating a cone disturbance (Fig. 2B). In general, for families 30 and 225 the red threshold elevations lay below the green ones (Fig. 2B), whereas in families 1935 and 479 red and green threshold elevations overlapped, with the loss for each being similar (Fig. 2B-the solid line represents the range of the green readings). This is more clearly emphasised in Fig. 2C, where predominantly high G-R index values were obtained for

RED
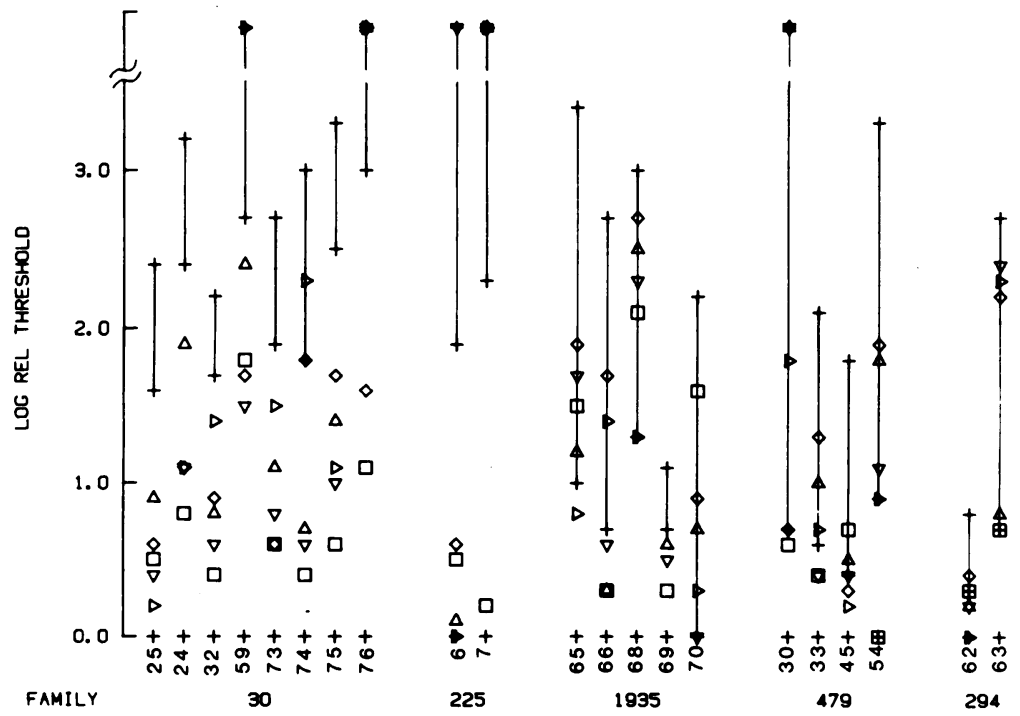

Fig. 2B Log relative thresholds for the red target in families 30, 225, 1935, and 479. The lines are those of Fig. $2 A$ and show the spread of readings with the green target. The symbols show the readings with the red target and have the meanings defined in Fig. 1 (lower). 
Fig. 2C G-R index for families 30, 225,1935 , and 479 . Filled symbols represent those index values associated with a threshold elevation for the red target of $<1$ log unit.

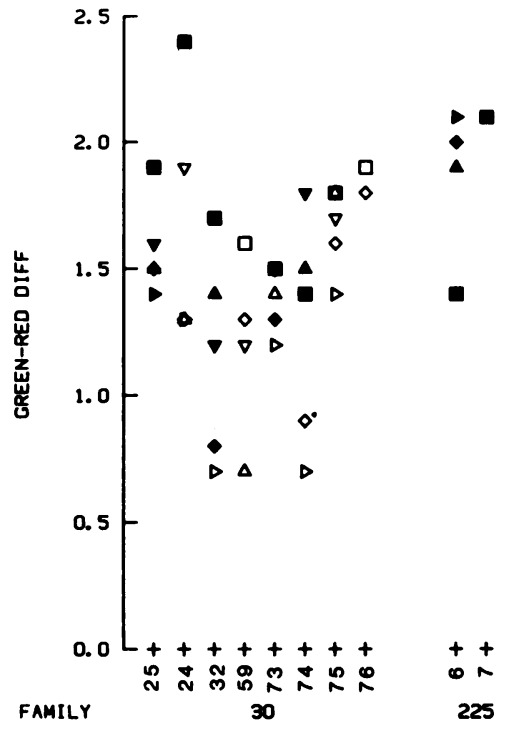

INDEX

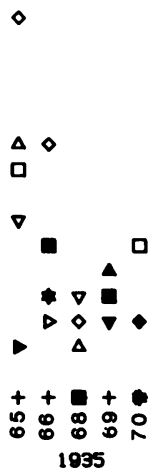

families 30 and 225 , indicating a much greater disturbance of rod function than cone function, and generally lower index values were present in families 479 and 1935, indicating that rod and cone losses were similar within a given area. However, there was an overlap of index values between the two sets of families, and low values in family 30 indicated that, though rod thresholds might be highly elevated, rod function was present. Absence of rod involvement in the detection of the green target requires an index value of about $2 .^{7}$ A distinguishing feature is that, where cone function was near normal (indicated by the filled symbols), the index reached values of 1.5 or more in some zones for families 30 and 225 but was always less for families 479 and 1935 .

We have derived criteria for classifying patients

GREEN

Fig. 3A Log relative thresholds for the green target in families 1188 , 675, and 9999. See legend to

Fig. $2 A$.

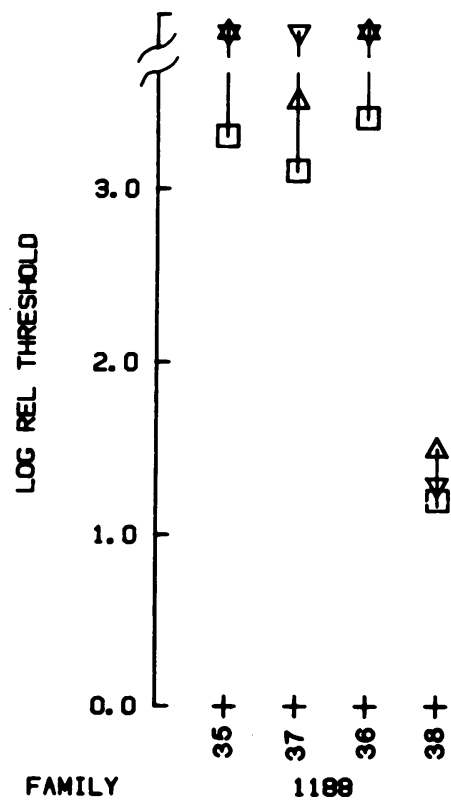

1180
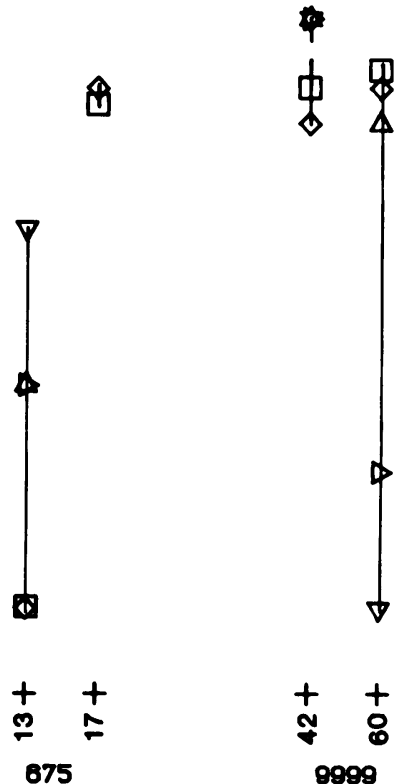

9999 
from the features seen in Fig. 2. The criteria for a diffuse and selective pattern of rod loss, pattern $D$, are: (1) in all zones threshold elevations for the green target must be greater than 1.5 log units; (2) in at least one zone the elevation for the red target must be less than $1 \log$ unit, and this must be accompanied by an index value of greater than 1.5. The diffuse description applies only to loss of rod function; loss of cone function may be regional, as in family 225 . The criteria for a regional loss of both rod and cone function, pattern $R$, are: (1) in at least one zone threshold elevations for both red and green targets must be less than 1 log unit; (2) in at least one zone threshold elevations for the two targets must be greater than $0.5 \log$ unit. That these criteria are somewhat arbitrary can be seen in family 30 (Fig. 2B): two patients, $30 / 59$ and $30 / 76$, did not show any zone where threshold elevations for the red target were less than $1 \log$ unit, even though the general pattern of their data was in line with other members of the family. Similarly, patient $1935 / 68$ failed to conform to the strict pattern $\mathrm{R}$ criteria because in no zone did the threshold elevation for both targets fall below 1 log unit. In these three cases from large families, and one other from a family of 8 , we have relaxed the criteria and classified the individuals according to the family pattern. However, where such family information has not been available, we have not classified any patients who did not fulfil the criteria for either pattern. Thirteen individuals from four families showed pattern $\mathrm{D}$ behaviour, 28 from 13 families pattern $R$; the remaining 63 were unclassifiable and are referred to as $U$ patients. Nineteen of the $\mathrm{U}$ patients had Goldmann fields of $5^{\circ}$ or less for the I/4 target and gave 'off scale' threshold readings for both static perimetry targets outside a $5^{\circ}$ eccentricity.

Figs. 3A, B, C show results from three families illustrating some of the problems in classification. Although patients $1188 / 35,1188 / 36$, and $1188 / 37$ had severe and diffuse loss of rod function, this was not the case with patient $1188 / 38$, who showed a mild rod loss accompanied by near normal cone function at the tested locations. No member of the family fulfilled the criteria for either pattern D or R, and they were all placed in the U category.

In families 675 and 9999 patients $675 / 17$ and 9999/42 showed severe and diffuse loss of rod function with better preservation of cone function (Fig. 3), and it might be thought they were similar to pattern $D$ patients. However, the other family member in each case clearly conformed to pattern $R$ criteria. It is plausible to suggest that the regional pattern is lost as disease progresses; both patients 675/17 and 9999/42 had limited fields, and cone function, where preserved, was severely disturbed. In the absence of further information they have both been placed in the U category. This example emphasises the importance 
Fig. 3C $G$-R index for families 1188,675 , and 9999 . See legend to Fig. $2 C$.

\section{INDEX}

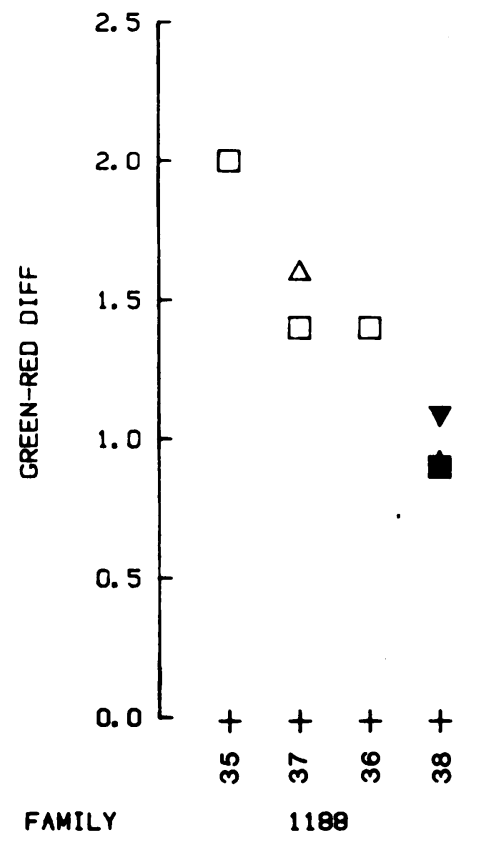

D patients belong to the A subgroup and all but two of the pattern $R$ patients to the $B$ subgroup (the two exceptions belong to the $\mathrm{A} 2$ subdivision - that is, no rod ERG but rod psychophysics). However, subgroups $\mathrm{A}$ and $\mathrm{B}$ also include $\mathrm{U}$ patients who have not been classified by static perimetry.

Fig. 4 summarises some of the ERG data. It consists of histograms showing how many of the patients in each of the subgroups $D, R$, and $U$ produced a $b$ wave in a given amplitude or latency range for a particular stimulus. Results are shown for two stimuli: (1) a blue flash sufficiently bright to elicit a saturated rod response in normal observers but not bright enough to yield a significant contribution from the cone system; (2) a red flash which in normal observers separates out cone (a1 and b1 waves) and rod components ( $\mathrm{a} 2$ and $\mathrm{b} 2$ waves) and produces saturated cone ones; the data shown refer to the cone b1 waves. The range label $\mathrm{N}$ refers to responses falling within normal limits (see above) and the labels $\mathrm{A}^{+}, \mathrm{A}^{++}, \mathrm{A}^{+++}$refer to increasing degrees of abnormality (see legend to Fig. 4). The ND category refers to the cases where measurements were not done.

The amplitude information (Fig. 4, left side) shows that, for the blue flash, all $D$ patients fell into the $\mathrm{A}^{+++}$and $\mathrm{A}^{++}$category (the small responses here being due to the cone system), while all but three of the $\mathrm{R}$ patients fell into the $\mathrm{A}^{+}$and $\mathrm{N}$ categories. On the other hand for the red flash the difference 


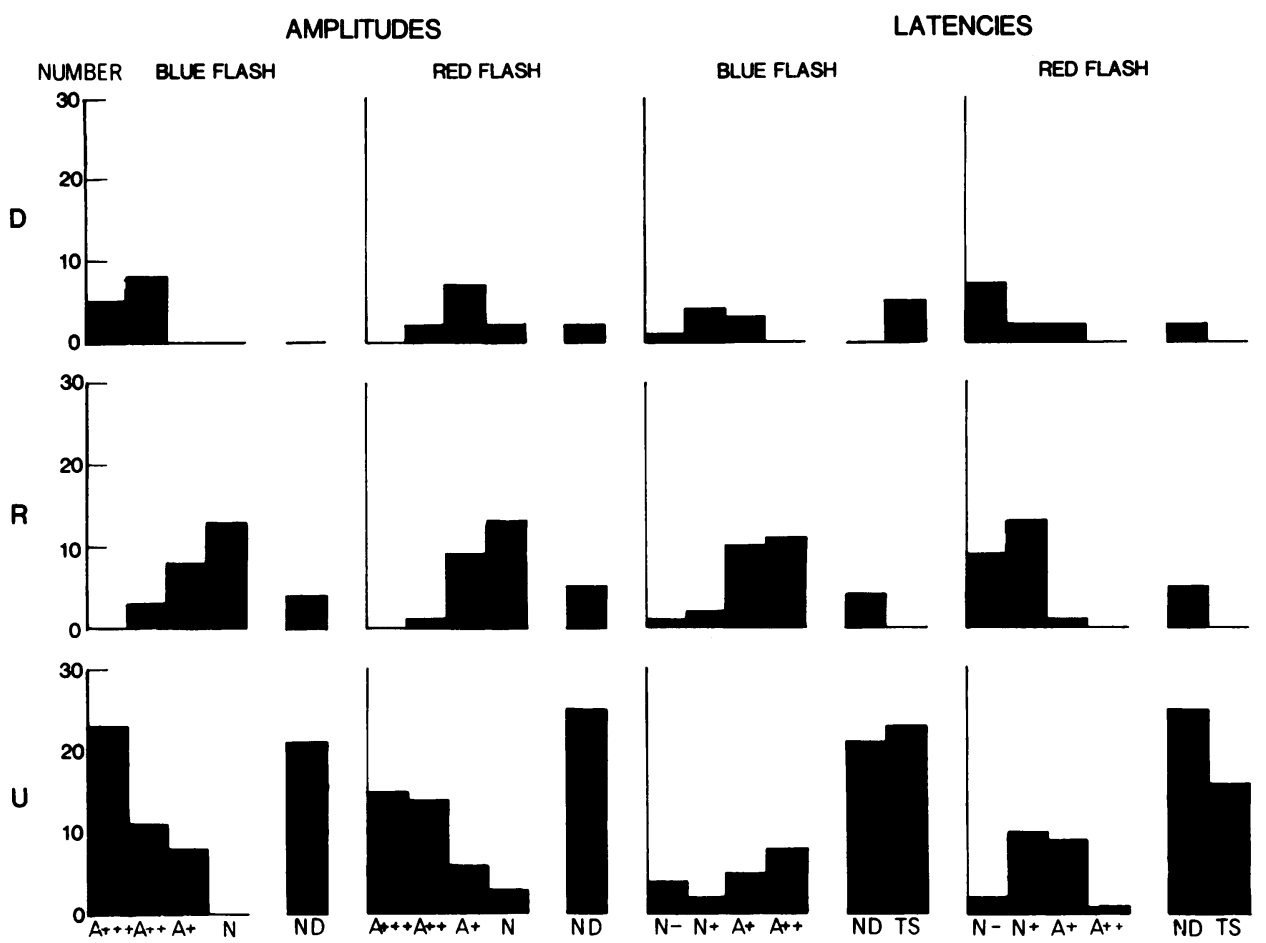

Fig. 4 Distribution of ERG amplitudes and latencies for the AD RP subgroups. D: subgroup with diffuse rod loss; $R$ : subgroup with regional receptor loss; $U$ : unclassified. Blue flash refers to run 12 of Arden et al., ${ }^{8}$ red flash to run 18. ' $R e d$ flash' amplitudes and latencies refer to cone $b$ wave. $N=$ within 2 standard deviations of normal mean. $A+, A++$, etc. $=$ increasingly abnormal. $N D=$ not done. $T S=$ too small to measure. For 'blue flash' amplitudes $N=>140 \mu V$; $A+=20-140 \mu V ; A++=5-19 \mu V ; A+++=<5 \mu V ;$ mean normal amplitude $=319 \mu V$. For 'red flash' amplitudes $N=>59 \mu V ; A+=20-59 \mu V ; A++=5-20 \mu V ; A+++=<5 \mu V ;$ mean normal amplitude $=153 \mu V$. For 'blue flash' latencies $N-=<57 \mathrm{~ms} ; N+=57-69 \mathrm{~ms} ; A+=70-79 \mathrm{~ms} ; A++=>79 \mathrm{~ms}$; mean normal latency $=57 \mathrm{~ms}$. For 'red flash' latencies $N-=<47 \mathrm{~ms} ; N+=47-57 \mathrm{~ms} ; A+=57-69 \mathrm{~ms} ; A++=>69 \mathrm{~ms}$; mean normal latency $=47 \mathrm{~ms}$.

between the groups disappeared: the majority of both $\mathrm{D}$ and $\mathrm{R}$ patients fell into the $\mathrm{A}^{+}$and $\mathrm{N}$ categories. This is expected, because assigning an individual to either group requires near normal cone function in some parts of his or her visual field. Likewise the preponderance of $U$ patients in the $\mathrm{A}^{+++}$and $\mathrm{A}^{++}$categories for both blue and red flashes is expected because they have very poor rod and cone function.

As regards the latency distributions (Fig. 4, right side), the blue flash values for the $D$ patients are not informative, since they are derived from cone responses and the norms are for rod $b$ waves. For the $R$ patients most of the values were abnormal. The complete amplitude-intensity functions for these patients show that they responded as if the stimulus intensity had been reduced (see Arden et al $^{6}$ ). Since latencies are more sensitive to changes in effective intensity over this range, this would explain, at least in part, how amplitudes in the normal range can go hand in hand with abnormal latencies. The high proportion of nearly normal latencies for the red flash responses in both $\mathrm{D}$ and $\mathrm{R}$ groups again reflected the bias introduced by the classification procedure. Interestingly, in some members of the $\mathrm{D}$ group a normal latency for the red flash b1 wave was accompanied by abnormal timing in the responses to very bright white light flickering at $30 \mathrm{~Hz}$ (run 31 , Arden et $\left.a l .{ }^{8}\right)$; the reason for the discrepancy is not clear. None of the $\mathrm{D}$ or $\mathrm{R}$ patients, so far as we are aware, belong to autosomal dominant families with incomplete penetrance. In the case of the $U$ group most of the responses were too small, if they existed at all (TS category), for latency measurement and the few values in the normal range seen for the blue flash resulted from an inappropriate comparison of cone ERGs with rod norms, as in the case of the D patients.

Arden et al. ${ }^{6}$ have attempted to predict ERG amplitude versus log intensity functions from psychophysical data on the basis of a model with a number of simple assumptions. In the case of data from D 
Fig. 5 Distribution of ages at examination, ages at onset of night blindness, and ages at onset of field loss for the AD RP subgroups.
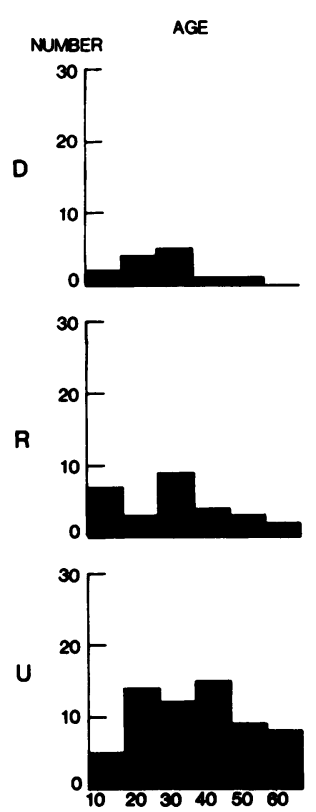
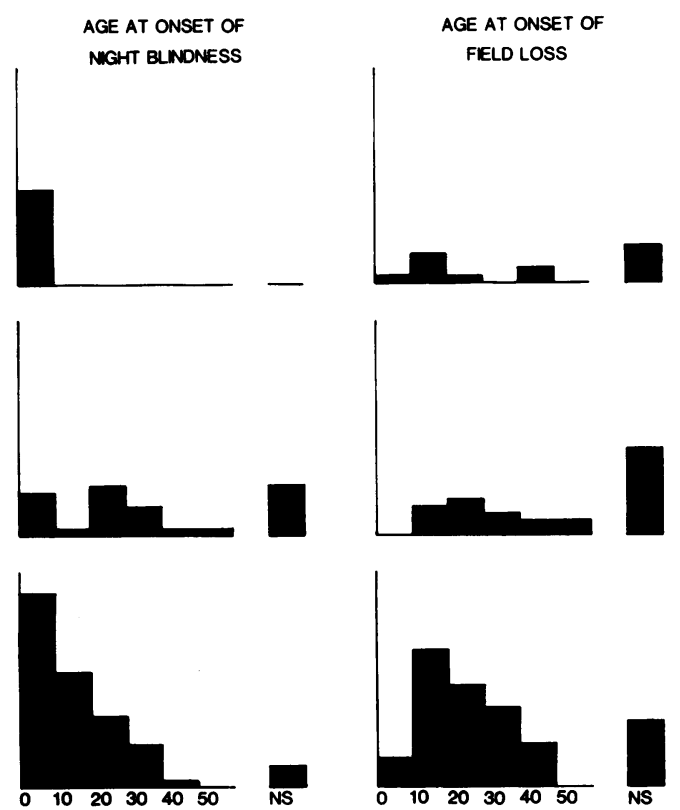

patients analysed this way the model successfully accounted for the absence of measurable rod ERGs. In the case of $10 \mathrm{R}$ patients (the data from the remaining 18 were not available) the model gave good fits to the ERG data in four cases, but in six the actual loss in ERG sensitivity as measured by the shift of the amplitude versus log intensity function along the log intensity axis was much smaller than predicted from the psychophysical results. Moreover, the maximum b wave amplitudes were smaller than expected.

\section{SYMPTOMS}

Fig. 5 summarises (1) the distribution of ages among patients in the three groups, D, R, and U; (2) the distribution of ages at which they first became aware of problems in the dark; and (3) the distribution of ages at which they first remembered having visual field problems. (NS refers to those patients claiming no symptoms). All D patients reported night blindness before the age of 10 and none were asymptomatic. By contrast, the majority of $R$ patients reported problems occurring after the age of 20 , or were asymptomatic. Of the seven exceptions, in two cases there was definite reason to doubt the usefulness of the patients' reports, for the functional state of their rods, as judged from the static perimetry and ERG data, was not severely disturbed and not very different from that of other members of their families who all reported late onset. In the other cases, especially in two of them who lacked rod ERGs, functional losses were more consistent with the patients' reports of early onset. In the $U$ group the majority reported early onset.

There is little significant difference between the $D$ and $R$ groups in age of onset of field loss. In the $R$ group there was a tendency for patients to report awareness of field losses and night blindness at the same time. The two events were separated by five years or less in nine of the 13 patients who reported both symptoms. In the $\mathrm{D}$ group the separation was less than five years in only two patients, while, in six of the remaining 11 in the group the separation was more than 20 years or else field loss was not reported, even though night blindness had been experienced more than 20 years earlier. The $U$ group showed a distribution skewed to early field losses, which is consistent with the small fields seen in most of them at the time of their examination.

VISUAL ACUITY

Table 3 summarises the distribution of visual acuities among the patient groups. All but two of the D and R patients had acuities better than or equal to $6 / 12$. In

Table 3 Distribution of visual acuities in the groups of AD RP

\begin{tabular}{llll}
\hline Visual acuity & Number of $D$ & Number of $R$ & Number of $U$ \\
\hline$>6 / 12$ & 8 & 24 & 21 \\
$=6 / 12$ & 4 & 3 & 12 \\
$<6 / 12$ & 1 & 1 & 28 \\
No information & 0 & 0 & 2 \\
\hline
\end{tabular}




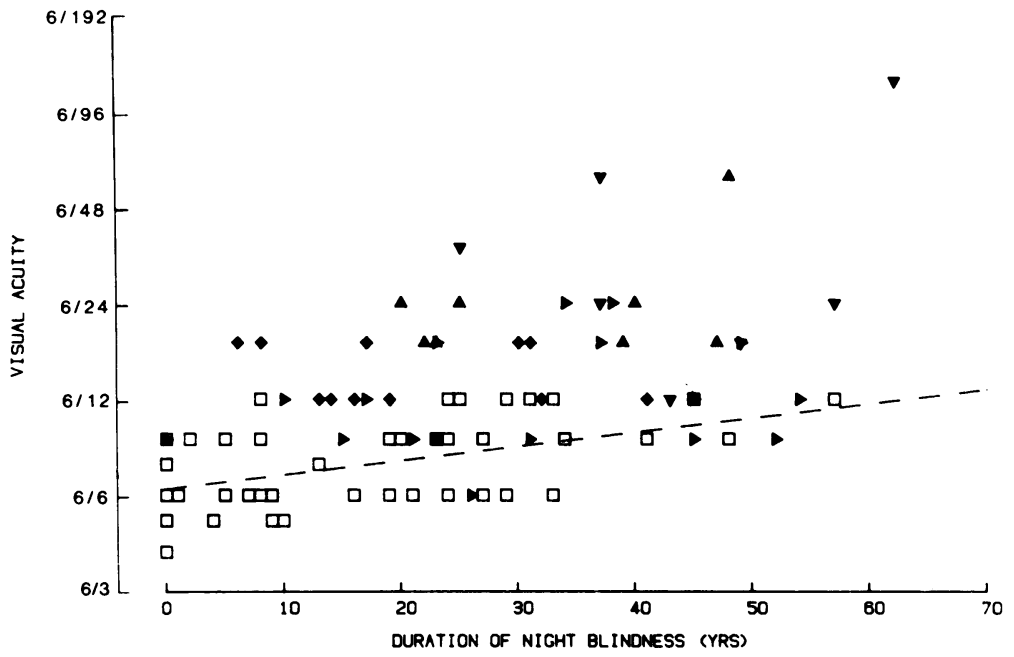

the $U$ group about $46 \%$ had acuities less than $6 / 12$; this probably reflects the selection bias introduced by the method of classification.

Any patient with a visual acuity of less than 6/12 had either macular oedema or macular atrophy, and/or lens opacities. Decreased acuity at an early age or after a short duration of night blindness was due to macular oedema, except in one patient aged 55 years who had age-related cortical lens opacities. All but one of the patients with cataract had posterior subcapsular lens opacities; three also had nuclear sclerosis, and only one had peripheral cortical opacities.

A more detailed analysis of the dependence of visual acuity on disease duration and lens and macular problems was carried out with the help of the statistical package, MINITAB, which treated $\log _{2}$ acuity as a linear function of a series of explanatory variables and performed stepwise regression on the data. The following information was entered for each patient: (1) visual acuity expressed as a $\log _{2}$ ratio; (2) age at time of examination; (3) duration of symptoms of nightblindness; (4) duration of symptoms of field loss; (5) the presence (scored as 1 ) or absence (scored as 0 ) of lens opacities; (6) the presence or absence of macular oedema; (7) the presence or absence of macular atrophy. Full data were available on 93 patients. For this analysis no distinction was made between the subgroups. The most economic description of $\log _{2}$ VA was in terms of a linear model with five explanatory variables, namely: (1) night blindness duration; (2) lens opacities without macular problems; (3) macular oedema without lens opacities; (4) macular oedema with lens opacities; (5) macular atrophy with lens opacities. The model states that for every year of night blindness visual
Fig.6 $\log _{2}$ visual acuity as a function of duration of night blindness. Line is derived from a stepwise linear regression (Minitab) involving five explanatory variables, varl =duration of night blindness; var $2=$ presence (right-pointing triangles) or absence of lens opacities; var3=presence (diamonds) or absence of macular oedema; var4=presence $($ upward-pointing triangles) or absence of both lens opacities and macular oedema; var $5=$ presence

(downward-pointing triangles) or absence of both lens opacities and macular atrophy; unfilled squares indicate cases with no lens or macular problem. The model used:

$\log _{2} V A=0.0664+0.0147^{*}$ varI $+0.49^{*}$ var $2+0 \cdot 81^{*}$ var $3+1.48^{*}$ var 4 $+1 \cdot 60^{*}$ var5.

$R^{2}=68 \%$; mean square residual $=(0.495)^{2} ;$ number of cases $=93$; degrees of freedom $=87$.

acuity is reduced by a factor of 1.01 ; a score of 1 for one of the other variables leads to a further independent reduction by a factor of 1.40 for a lens opacity, 1.75 for macular oedema, 2.79 for the combination of lens opacity and macular oedema, and 3.03 for a combination of lens opacity and macular atrophy. Fig. 6 shows the visual acuity data on a $\log _{2}$ scale plotted as a function of duration of night blindness, with other factors indicated by various filled symbols. The dashed line is the model's prediction of the effect of the night blindness duration alone and provides a reasonable fit to the unfilled squares, which represent data from patients without macular or lens complications.

An almost equally satisfactory model can be set up by replacing duration of night blindness by duration of field loss symptoms, but substitution of age for either of the duration variables leads to a poorer fit. Since the three variables are correlated (the correlation coefficient between the duration variables is 0.78 and between age and night blindness duration 0.67), only one of them needs to be represented in the model. Thus our data provide no evidence of any effects of age per se which are not accounted for by the duration of the disease or a lens or a macular problem.

\section{GOLDMANN FIELDS}

In most patients the area of upper visual field, as measured by the Goldmann field target IV/4, was considerably smaller than the area of the lower field; 


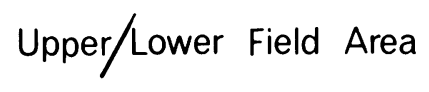

\section{NUMBER}
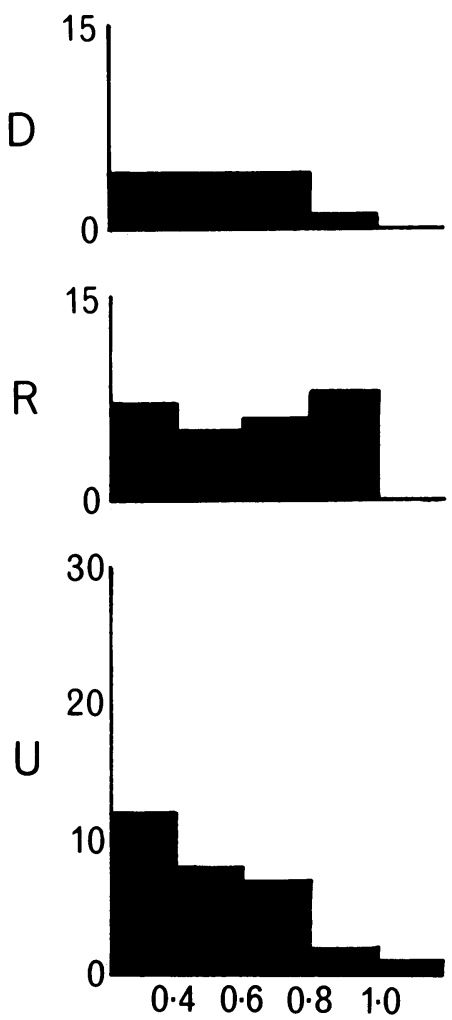

Fig. 7 Distribution of field area ratios (upper/lower) for the $A D$ RP subgroups. $V S F=$ very small fields. $N D=$ not done. the ratio of the two areas was typically smaller than the normal ratio of $0 \cdot 9$. Fig. 7 shows the distribution of ratios for the three patient groups (VSF refers to those patients with very small fields where the ratio is not meaningful and ND to those cases where the fields were not done). Full or partial ring scotomas were seen in all groups but the numbers in each were very small. Qualitatively the appearance of fields was similar for the two eyes in most of the patients.

In general the area of the visual fields was related to the length of time visual field problems had been experienced. Fig. 8 shows $\log _{\mathrm{e}}$ of the field area in $\mathrm{cm}^{2}$ enclosed by the contour for the IV/4 target, plotted as a function of duration of field loss. The results from the three goups have not been distinguished, since most of the $D$ and $R$ patients have experienced field loss symptoms for less than 10 years.

The combined field area scores together with the information used in the analysis of visual acuity were analysed by a stepwise linear regression procedure. The dependent variable, $\log _{e}$ field area, can be represented as a linear function of two explanatory variables, namely (1) duration of field loss and (2) the presence of both a lens opacity and macular oedema. If all the data are considered, the model predicts that for every year of noticeable field loss there is a decline in field area by a factor of 1.09 (dashed line of Fig. 8). However, the line is biased by a point obtained from a patient with 59 years of field loss who had an unusually large area from an island of vision surviving in his inferior field. If this point is excluded, the model predicts a decline by a factor of 1.11 per year of field loss (solid line). The presence of an opacity together with macular oedema (indicated by the circled points) independently reduces field area by a

Fig. $8 \log _{e}$ field area as a function of duration of field loss. Lines derived from stepwise linear regression (Minitab) involving two explanatory variables, varl $=$ duration of field loss, var2 = presence (circled points) or absence of both lens opacities and macular oedema. The models used: line):

(1) all points included (dashed

$\log _{\text {e }}$ field area $=7 \cdot 14-0 \cdot 0882^{*}$ varl$2 \cdot 00^{*}$ var 2 .

$R^{2}=55 \cdot 8 \%$, mean square

residual $=(1 \cdot 23)^{2}$; number of

cases $=90 ;$ degrees of freedom $=87$

(2) One extreme point excluded-see text (solid line):

$\log _{\text {e }}$ ield area $=7 \cdot 19-0 \cdot 105^{*}$ varl $-1 \cdot 83^{*}$ var2.

$R^{2}=63 \cdot 3 \%$; mean square residual $=(1 \cdot 22)^{2}$; number of cases $=89 ;$ degrees of freedom $=86$

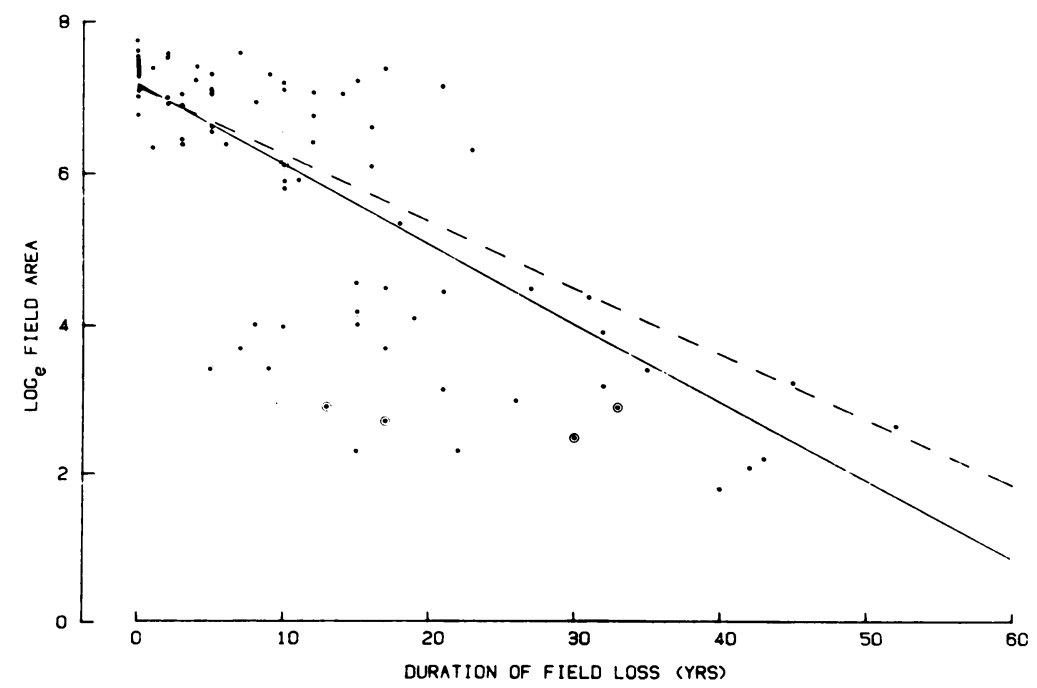


further factor of $6 \cdot 23$. However, it is clear that the model can give only a very approximate description of the field area data. It should be remembered that the analysis has two major weaknesses-namely, the duration of field loss is a subjective variable, and the analysis is based on a cross-patient comparison of a group which is not likely to be homogeneous.

\section{APPEARANCE OF FUNDUS}

Most of the patients had attenuated retinal vessels and pale discs. Early change was seen as a greying of the pigment epithelium. With later migration of pigment into the retina the pigment epithelium assumed an atrophic appearance. The area of maximum involvement was usually, though not exclusively, the mid periphery, with a zone anterior and posterior to this which showed lesser degrees of pigment epithelial change. The area within $10^{\circ}$ radius from fixation was usually uninvolved, unless macular oedema or atrophy was present. As might be expected for progressive disease, a sharp cut-off is not seen. The degree of pigment migration into the retina was greater the longer the duration of the disease and the greater the age of the patient. The area of maximum change in the fundus agreed well with the area of field loss measured with the Goldmann IV/4 target. Thus changes in the inferior compared with superior retina were generally more prominent. This might be due to the effect of more illumination on the inferior retina or the result of intrinsic retinal factors. On examination of the fundi no differentiating features could be found between $D$ and $R$ patients.

\section{Discussion}

\section{NATURE OF THE SAMPLE}

Most previous studies of RP have been carried out on small numbers of patients, often with several genetic groups in a sample. In the present study we have concentrated on a single genetic group and have examined over 100 patients with AD RP. The fact that we had to call for volunteers for lengthy examinations during the working week has introduced some bias: there is an excess of females and of patients in the 20-50 age group (Table 1). Further, in order not to restrict our sample unduly we have included families in which a diagnosis of AD RP is very probable but the confirmatory evidence of male to male transmission is absent. Such families account for just over $50 \%$ of our patients. However, given the total number examined, these factors should not strongly bias the survey.

PROGNOSIS

It is commonly stated that the prognosis for visual acuity (VA) is better in AD RP than in AR and X-L disease, ${ }^{911}$ though this has been disputed." About $60 \%$ of our patients have a VA better than or equal to $6 / 12$ by the age of 50 , or after a duration of 40 to 50 years of the disease. This is a greater percentage than that quoted by Pearlman" and similar to that found by Jay and by Fishman. ${ }^{10}$

The factors associated with loss of visual acuity are (1) the presence of a secondary change such as a lens opacity, macular oedema, or macular atrophy (filled symbols of Fig. 6), and (2) duration of night blindness. Analysis of how these factors are interrelated reveals that with increasing duration of disease there is an increased probability of one or other of the secondary changes. However, there is also an independent, if small, effect of duration.

Macular oedema is more often associated with visual loss when the duration of disease is short, and macular atrophy when it is long. It may be that macular atrophy is a late consequence of macular oedema, but it is also possible that it is the result of longstanding disease. Certain families have a very high incidence of these macular changes, whereas others have none; this may represent a genetic variation.

To assess the effect of field loss on life we have assumed that a central field of radius $10^{\circ}$ with the IV $/ 4$ target represents the minimum field required by a patient to be independently mobile. In our sample $93 \%$ of the patients in the under- 20 age group, $89 \%$ in the $20-40$ age group, and $60 \%$ in the over 40 age group had a field greater than or equal to this.

\section{CLASSIFICATION}

From an examination of intrafamilial and interfamilial differences in the static perimetry data obtained from large families we have identified two subgroups of AD RP: in one there is severe and diffuse loss of rod function which in some regions coexists with relatively normal cone function; in the other loss of rod function is regional and accompanied by loss of cone function. We have designated the pattern of behaviour of the first subgroup by the letter $\mathrm{D}$ (which stands for diffuse loss of rod function) and the second by the letter $\mathrm{R}$ (which stands for regional loss of rod function). The letter $U$ refers to those patients whom we have not placed in subgroups $\mathrm{D}$ or $\mathrm{R}$. The classification depends on measurements of the state of rod function by static perimetry and not on observations of fundus appearance or Goldmann fields. These do not distinguish between the subgroups, both of which show regional pigmentation and regional cone losses.

With ERG measurements a very similar subgrouping could have been obtained independently. D patients do not have a rod a or b wave, but most do 
have a maximum cone $\mathrm{b}$ wave greater than $20 \mu \mathrm{V}$. Most $R$ patients have both maximum rod and cone $b$ waves greater than $20 \mu \mathrm{V}$. Only two cases in each of the subgroups classified by static perimetry would not meet these criteria.

The patients of Arden et al. ${ }^{6}$ are included in the present survey, but the method of classification in the previous study was based solely on the presence or absence of the rod ERG: if absent, patients were placed in subgroup $A$, if present, in subgroup $B$. This means that $A$ includes not only $D$ patients with a sizeable cone ERG, but also those patients who do not have any ERG at all (U patients). The equivalence of the $B$ and $R$ subgroups is closer, though not exact. Two $R$ patients have no rod ERGs and were classified by Arden et al. ${ }^{6}$ as 'A2.'

The age of onset of night blindness is not a reliable indicator of $D$ or $R$ patterns, since onset before the age of 10 was reported not only by all $D$ patients but also by a substantial number of $U$ and even a few $R$ patients. There is a better but far from exact correspondence between the $R$ pattern of behaviour and onset after 20 years of age.

The distinctions between patterns $\mathrm{D}$ and $\mathrm{R}$ may arise in three possible ways: (1) they are different stages along a progressive course of deterioration; (2) they are different phenotypic expressions of the same basic gene defect; (3) they represent different gene mutations. The general constancy of a pattern of visual loss within large families over a wide range of ages supports the hypothesis of genetic heterogeneity.

Massof and Finkelstein ${ }^{34}$ first proposed the distinction between severe and diffuse rod disease (type 1) and regional photoreceptor disease (type 2 ). Broadly our $\mathrm{D}$ patients conform to the description of type 1 and our $\mathbf{R}$ patients to type 2 . However, there are some differences: a feature emphasised for type 1 is that the degree to which rod losses are greater than cone losses is a constant over the whole visual field. In terms of our data this would mean very little variation in the G-R index for different zones. This is not the case for our D patients (Fig. 2C). For some patients the index falls to values of less than 1 in some regions. This indicates that there is still some rod function left in these regions and that cone losses are 'catching up' with rod losses. Another difference is that for type 2 the characteristic region of loss is the mid periphery. Whilst this is true for most of our $\mathbf{R}$ families, in two the main region of loss is far-peripheral (e.g. see Fig. 10 in Ernst et al. $\left.{ }^{7}\right)$. In view of these differences and the possibility that AD RP consists of more than two disease forms it is probably not appropriate at this stage to make a complete identification between $\mathrm{D}$ and type 1 subgroups on the one hand, and $R$ and type 2 on the other.
The D families appear to form a more homogeneous subgroup than the $\mathrm{R}$ ones. Variation in the region maximally affected by disease may indicate that the $\mathbf{R}$ families need to be further subdivided. Yet another pointer to possible heterogeneity in the $R$ subgroup is the variation in rod ERG. In the $R$ group there are three individuals from three families who produce ERGs of less than $20 \mu \mathrm{V}$ to the blue flash, whereas $R$ patients typically give more than $40 \mu \mathrm{V}$ and many fall within the normal range (greater than $140 \mu \mathrm{V})$. Two of these patients report early onset of night blindness, and the other family members are severely affected and in the $U$ category. More information, especially that derived from longitudinal studies, is needed to determine the basis of this heterogeneity.

Almost all our $\mathrm{D}$ and $\mathrm{R}$ patients were classified on an individual basis, though four exceptions were made in the case of three large families, where the characteristics of other family members were taken into account. However, in the case of small family units, such as families 675 and 9999 illustrated in Fig. 3 , where an individual does not conform to the criteria laid down for classification (e.g., 675/17 and 9999/42) he or she has been assigned to the U category, even though his or her relative is placed in one of the subgroups (e.g., 675/13 and 9999/60) in the $R$ subgroup. If we were to classify these $U$ individuals according to the characteristics of their relatives, then the numbers in the D subgroup would rise from 13 to 16 and in the $R$ subgroup from 28 to 34 .

\section{DISEASE MECHANISMS}

Can we infer anything about the nature of the disease process at the cellular level for the two subgroups? The key feature of pattern $D$ is the combination of very poor rod function throughout the retina with near normal cone function in some regions. This suggests that the major problem is in the rod system. The absence of any measurable rod ERG, including an a wave produced by the rods themselves, together with a relatively well preserved cone ERG, points to a defect in the rod transduction mechanism prior to the generation of the a wave. It is tempting to see the widespread rod disturbance as the primary condition of the disease with the progressive deterioration of the cones as a secondary phenomenon. This would explain why night blindness is perceived from infancy while major field losses are only apparent later in life, and why the pigmentation of the fundus has a regional appearance and is characteristic of late disease. Nevertheless our results do not exclude the possibilities that rod losses are regional early in life but become diffuse with time, that regional cone losses are part of the primary disease mechanism, and that cone abnormalities are occurring in areas where 
cone thresholds are normal. Our view of a primary rod transduction defect throughout the retina has yet 'to be tested.

In the $\mathrm{R}$ patients the disease appears to be affecting both types of receptor in a given region-sometimes more or less equally as judged by threshold behaviour (e.g., see Fig. 10, Ernst et al. ${ }^{7}$ ) and sometimes with the rods more severely affected (e.g., see 675/17 and 9999/42, Fig. 3). A comparison of the ERG and psychophysical data (see Arden et al. ${ }^{6}$ ) points to a disturbance of not only photoreceptors but also of postsynaptic layers. There is also evidence that the disease is not confined to retinal cells. $R$ patients show an elevated osmotic fragility of their erythrocytes, whereas the fragility of the red blood cells of D patients is normal. ${ }^{12}$

Future advances in research on AD RP will come from the application of new tests such as fundus reflectometry to patients already investigated by the techniques described in this study, longitudinal studies, the investigation of young family members, and histopathological and biochemical studies from donor eyes of properly characterised patients. From such studies it should become clearer whether our attempt at classifying AD RP has a valid basis, whether it needs to be refined and extended, and what are the underlying pathogenetic mechanisms.

We gratefully acknowledge financial support from the Medical Research Council, the British Retinitis Pigmentosa Society, and the US National Retinitis Pigmentosa Foundation. We thank Mr C R Hogg, Mr G Joseph, Mr G Mould, and Mrs K Shah for technical assistance, Dr D J Powell and Miss S Wooding for computing assistance, Mrs V Hanna and Miss M Javeleau for secretarial help, and $\mathrm{Mr} \mathrm{P}$ Clarke for carrying out the statistical analysis. We also thank Dr M Jay for help with genetic advice and Dr G Fishman for discussion of ERG data. Throughout the project we have received encouragement and advice from Mr B Jay. Finally we would especially like to thank all the patients who so willingly took part in the study.

\section{References}

1 Krill AE. Retinitis pigmentosa: a review. Sight Sav Rev 1972; 42: 21-8.

2 Berson EL, Gouras P, Gunkel RD, Myrianthopoulos NC. Dominant retinitis pigmentosa with reduced penetrance. Arch Ophthalmol 1969; 81: 226-34.

3 Massof RW, Finkelstein D. Rod sensitivity relative to cone sensitivity in retinitis pigmentosa. Invest Ophthalmol Visual Sci 1979; 18: 263-72.

4 Massof RW, Finkelstein D. Two forms of autosomal dominant retinitis pigmentosa. Doc Ophthalmol 1981; 51: 289-346.

5 Marmor MF. The electroretinogram in retinitis pigmentosa. Arch Ophthalmol 1979; 97: 1300-4.

6 Arden GB, Carter RM, Hogg CR, et al. Rod and cone activity in patients with dominantly inherited retinitis pigmentosa: comparisons between psychophysical and electroretinographic measurements. BrJ Ophthalmol 1983; 67: 405-18.

7 Ernst W, Faulkner DJ, Hogg CR, Powell DJ, Arden GB, Vaegan. An automated static perimeter/adaptometer using light emitting diodes. BrJ Ophthalmol 1983; 67: 431-42.

8 Arden GB, Carter RM, Hogg CR, et al. A modified ERG technique and the results obtained in $\mathrm{X}$-linked retinitis pigmentosa. Br J Ophthalmol 1983; 67: 419-30.

9 Jay B. Hereditary aspects of pigmentary retinopathy. Trans Ophthalmol Soc UK 1972; 92: 173-8.

10 Fishman GA. Retinitis pigmentosa: visual loss. Arch Ophthalmol 1978; 96: 1185-8.

11 Pearlman JT. Mathematical models of retinitis pigmentosa: a study of the rate of progress in the different genetic forms. Trans Am Ophthalmol Soc 1979; 72: 643-56.

12 Hussain AA, Voaden MJ. Studies on retinitis pigmentosa in man. II. Erythrocytc osmotic fragility. BrJ Ophthalmol 1985; 69: $126-8$. 